\title{
Ginsenoside Rg3 Promotes Cell Growth Through Activation of mTORC1
}

\begin{abstract}
Wei Liu',2, Sheng-Xiong Zhang ${ }^{3}$, Bo Ai ${ }^{4}$, Hua-Feng Pan ${ }^{1}$, Dan Zhang ${ }^{1}$, Yu Jiang ${ }^{5}$, Lei-Hao Hu ${ }^{1,2}$, Ling-Ling Sun ${ }^{1,2 *}$, Zhe-Sheng Chen ${ }^{6 *}$ and Li-Zhu Lin ${ }^{1,2 *}$

${ }^{1}$ Lingnan Medical Research Center, Guangzhou University of Chinese Medicine, Guangzhou, China, ${ }^{2}$ Integrative Cancer Centre, The First Affiliated Hospital of Guangzhou, University of Chinese Medicine, Guangzhou, China, ${ }^{3}$ Guangdong Work Injury Rehabilitation Hospital, Guangzhou, China, ${ }^{4}$ Department of Thoracic Surgery, Tongji Medical College, Huazhong University of Science and Technology, Wuhan, China, ${ }^{5}$ Department of Pharmacology and Chemical Biology, University of Pittsburgh School of Medicine, Pittsburgh, PA, United States, ${ }^{6}$ Institute for Biotechnology, St. John's University, Queens, NY, United States
\end{abstract}

OPEN ACCESS

Edited by:

Kenneth K. W. To,

The Chinese University of Hong Kong,

China

Reviewed by:

Sven Marcel Stefan,

University of Oslo, Norway

Xingqi Li,

Stony Brook University, United States

*Correspondence:

Ling-Ling Sun

sunlingling5094@gzucm.edu.cn

Zhe-Sheng Chen

chenz@stjohns.edu

Li-Zhu Lin

lizhulin26@yahoo.com

Specialty section:

This article was submitted to Molecular and Cellular Oncology,

a section of the journal

Frontiers in Cell and Developmental

Biology

Received: 24 June 2021

Accepted: 26 August 2021

Published: 13 September 2021

Citation:

Liu W, Zhang S-X, Ai B, Pan H-F, Zhang D, Jiang $Y$, Hu L-H, Sun L-L,

Chen Z-S and Lin L-Z (2021)

Ginsenoside Rg3 Promotes Cell

Growth Through Activation

of mTORC1.

Front. Cell Dev. Biol. 9:730309.

doi: 10.3389/fcell.2021.730309
Ginsenoside Rg3 is a steroidal saponin isolated from Panax ginseng. Previous studies have shown that Rg3 treatment downregulates the activity of rapamycin complex 1 (mTORC1) activity and inhibits the growth of cancer cells. However, the inhibitory effect of Rg3 on cancer cells is associated with high concentrations of Rg3 that are difficult to achieve in vivo. The human cervix adenocarcinoma HeLa cells were treated with Rg3. The protein levels of AMP-activated protein kinase alpha (AMPK $\alpha$ ), protein kinase $\mathrm{B}(\mathrm{Akt})$, ribosomal S6 protein(S6), and Erk were determined by immunoblotting analyses. We used a fluorescent probe to detect reactive oxygen species (ROS) production in living cells. The oxygen consumption rate (OCR) was examined by the Seahorse Extracellular Flux Analyzer. The content of adenosine triphosphate (ATP) was measured by ATPlite kit and Mitotracker was applied to detect the mitochondria. We showed that at lower concentrations, Rg3 activates mTORC1 independent of AKT and AMPactivated protein kinase (AMPK). Rg3 promotes mitochondrial biogenesis and function, increases the oxygen consumption of mitochondria and the content of ATP. This effect is in contrast to that of high concentrations of Rg3, which inhibits cell growth. These findings demonstrate a pro-growth activity of Rg3 that acts through mTORC1 and mitochondrial biogenesis and suggest a dose-dependent effect of Rg3 on tumor cell growth.

Keywords: ginsenoside Rg3, mTORC1, cell growth, cancer, Panax ginseng

\section{INTRODUCTION}

Rg3 is one of the pharmaceutical ingredients extracted from Panax ginseng. Its preparations have been widely used in cancer treatment to enhance patient's general health and improve the efficacy of chemotherapeutic agents (Lu et al., 2008; Zhou et al., 2016; Guo et al., 2018; Pan et al., 2019; Wang et al., 2019). A national survey on men and women in the United States estimated that $4-5 \%$ of those aged $45-64$ years used ginseng (Kaufman et al., 2002). Research to better understanding the therapeutic potential of Asian ginseng has been supported by the National Center for Complementary and alternative medicine (Jia et al., 2009). 


\section{Ginsenoside Rg3}
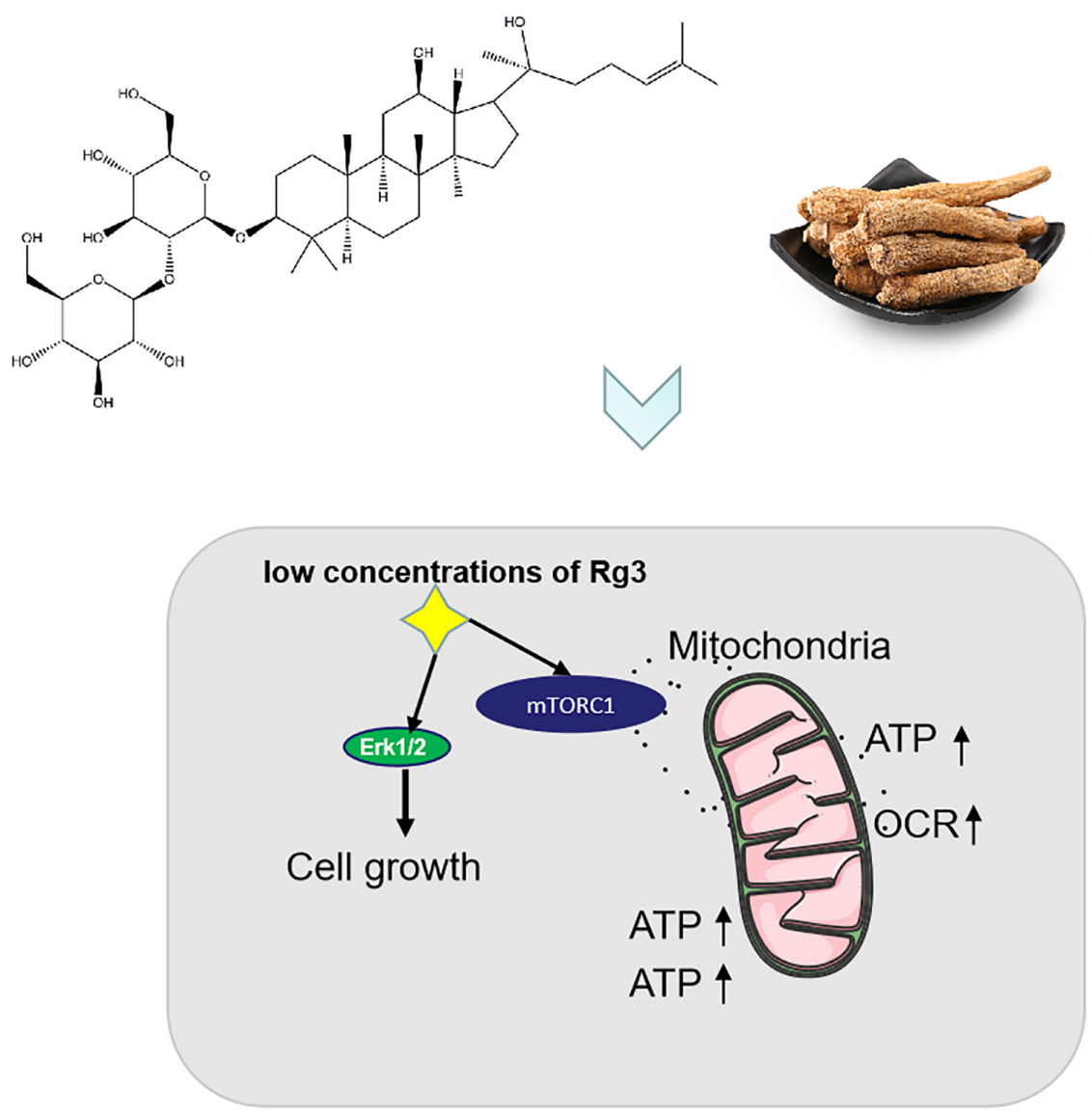

GRAPHICAL ABSTRACT | Ginsenoside Rg3 promotes cell growth through activation of mTORC1 and increasing mitochondrial biogenesis.

Previous studies have shown that Rg3 possesses activities to inhibit cell growth and induce apoptosis. It has been shown that Rg3 induces apoptosis via classical mitochondria-dependent caspase activation (Kim et al., 2013) and the death receptordependent extrinsic pathway (Kim et al., 2014). Rg3 exerts cytotoxic effects by activating the p53 signaling pathway and subsequently inducing apoptosis (Yuan et al., 2010; Zhang et al., 2015). In addition, $\operatorname{Rg} 3$ has been shown to inhibit mutant p53 and NF-кB signaling, possibly via the inactivation of extracellular signal-regulated kinase (ERK) and AKT to activate the mitochondrial cell death pathway (Kim et al., 2013, 2014; Aziz et al., 2016). Experiments studies also suggest that $\mathrm{Rg} 3$ in vivo and vitro can inhibit the growth of a variety of tumor cells both in vivo and vitro (Wang et al., 2014, 2018; Yuan et al., 2017; Chen et al., 2019). Previous studies have proposed that Rg3 inhibits cell growth by downregulation of lncRNA CCAT1 (Li and $\mathrm{Q} i, 2019)$, inhibition of $\mathrm{Wnt} / \beta$-Catenin, NF- $\kappa \mathrm{B}$, and mitogenactivated protein kinases (MAPK)/ERK signaling pathways (Kim et al., 2004; Lee et al., 2009; Yuan et al., 2010; He et al., 2011; Joo et al., 2015; Yang et al., 2016, 2017). These activities of Rg3 are believed to underlie the enhanced chemotherapeutic efficacy in clinical studies (Lu et al., 2008; Zhou et al., 2016) and in experimental mice (Chang et al., 2014; Shi et al., 2020). However, the pro-apoptosis and anti-proliferation effects of $\mathrm{Rg} 3$ occur only at high concentrations ( $>100 \mathrm{mM}$ ), which can be difficult to achieve in vivo due the low bioavailability of Rg3 (Xie et al., 2005; Jia et al., 2009).

In the present study we examined the effects of $\mathrm{Rg} 3$ on cell signaling and proliferation at low concentrations. We show that at at low concentrations, $\operatorname{Rg} 3$ activates the mTORC1 and promotes cell growth. This observation provides information on the efficacy of Rg3 and guide the selection of a safe dose for further study or human use as a certain in vivo concentration cannot be guaranteed.

\section{MATERIALS AND METHODS}

\section{Cell Line and Culture}

The human cervix adenocarcinoma HeLa cells obtained from the American Type Culture Collection (ATCC, Manassas, VA, United States) were cultured in EMEM supplemented with 10\% of fetal bovine serum (FBS) (Sigma-Aldrich Chemical Co., St. Louis, MO, United States). In order to inhibit the activity of 
mTORC1 and induce the baseline level of PS6 in HeLa cells, serum starved cells were cultured in serum starvation condition ( $0.5 \%$ FBS) for $24 \mathrm{~h}$ after cells reaching confluence. The cell cultures were incubated at $37^{\circ} \mathrm{C}$ in a humidified atmosphere with $5 \% \mathrm{CO}_{2}$. The cell cultures were incubated at $37^{\circ} \mathrm{C}$ in a humidified atmosphere with $5 \% \mathrm{CO}_{2}$.

\section{Cell Counting Kit-8 (CCK8 Assay)}

Cell growth was assessed using the Cell Counting Kit-8 (CCK8) assay. CCK8 assay was conducted in accordance with the manufacturer's instructions (BS350B, Biosharp, China). Briefly, HeLa cells $\left(1 \times 10^{3}\right.$ cells/well $)$ were seeded in 96-well plates. The next day, the cells were treated with $\mathrm{Rg} 3$ at various doses. After $24 \mathrm{~h}, 10 \mu \mathrm{l}$ of CCK8 solution was added to each well and incubated for $1 \mathrm{~h}$ before the light absorbance was measured at $450 \mathrm{~nm}$.

\section{Antibodies and Reagents}

The primary antibodies used in this study include the following: mouse anti-AMPK $\alpha$ (Cat. \# 2793), rabbit anti-phospho-AMPK $\alpha$ (T172) (Cat. \# 2535), rabbit anti-Akt (Cat. \# 9272), rabbit anti-phospho-Akt (Thr-308) (Cat. \# 4056), rabbit anti-phosphoAkt (S473) (Cat. \# 4060), mouse anti-S6 (Cat. \# 2317), phospho-S6(S235/236) (Cat. \# 2211), anti-Erk (Cat. \# 4695S), and anti-phospho-Erk (Cat. \# 9101S) were purchased from Cell Signaling Technology, mouse anti- $\beta$-actin (Cat. \# 612657) was purchased from BD Transduction Laboratories. Rg3 (Cat. \#SML0184, Sigma, purity >98\%) was dissolved in dimethyl sulfoxide (DMSO), The N-acetyl-l-cysteine (NAC) (Cat. \#194603, MP Biomedical) was dissolved in $\mathrm{H}_{2} \mathrm{O}$, Oligomycin (Cat. \# SLBZ3164, Sigma) and FCCP (Cat. \#C2920, Sigma), and tertButyl hydroperoxide (Cat. \#180340050, ACROS Organics).

\section{Western Blot Analysis}

Cells were lysed with lysis buffer. Samples were subjected to sodium dodecyl sulfate polyacrylamide gel (SDS-PAGE) electrophoresis. Western blot analysis was performed as described previously (Zhong et al., 2016) and the immobilized proteins were visualized by the enhanced chemiluminescent (ECL) detection system. Antibody concentrations were optimized with various dilutions to ensure that the blotting signals are linear to the levels of loaded proteins. Quantitative analysis of the blots was performed with densitometry scanning. Data from at least three independent experiments were analyzed.

\section{Reactive Oxygen Species Analysis}

The probe CellROX ${ }^{\circledR}$ Green Reagent (Molecular Probe, United States) was applied to detect the cellular reactive oxygen species (ROS) levels. Cells were treated with drug vehicle, Rg3, or $200 \mu \mathrm{M}$ of tert-butyl hydroperoxide as a positive control for $1 \mathrm{~h}$. Cells were stained with $5 \mu \mathrm{M}$ probe and $1 \mu \mathrm{g} / \mathrm{ml}$ DAPI for $30 \mathrm{~min}$ and then imaged on a laser scanning microscopy (LeicaTC-SP2 Confocal System) using a $40 \times$ objective.

\section{Measurement of Oxygen Consumption Rate}

HeLa cells were cultured on Seahorse XF 24 plates for 24 h. To detect oxygen consumption rate (OCR), the growth media was replaced with the XF Assay medium, and the plate was loaded into the Seahorse XF24 Analyzer. OCR baseline measurements were determined for HeLa cells pretreated with $\mathrm{Rg} 3$ or vehicle control for $24 \mathrm{~h}$.

\section{Measurement of ATP Content}

Cells were seeded in 96-well plates in triplicate and treated with $\operatorname{Rg} 3$ at various doses. After treatment for $24 \mathrm{~h}$, cells were measured with ATPlite kit (Perkin Elmer, United States), according to the instruction (Whitworth et al., 2012). All assays were performed with six replicates in three separate experiments and results are reported as the mean \pm SD.

\section{Mitochondrion Detection}

Live cells were incubated with $150 \mathrm{nM}$ Mitotracker Green FM (Life Technologies) for 30 min or with $100 \mathrm{nM}$ Mitotracker Red CMXRos (Life Technologies) for $40 \mathrm{~min}$ in the dark at $37^{\circ} \mathrm{C}$. Samples were then washed twice in PBS and imaged with a laser scanning microscope (LeicaTC-SP2 Confocal System). The relative fluorescence intensity was quantified by Image J software.

\section{Statistical Analysis}

All experiments were repeated at least three times and representative data were shown. All data were expressed as the mean $\pm \mathrm{SD}$. One-way analysis of variance (ANOVA) with multiple comparisons using Dunnett's test was applied to compare the differences amongst the groups. $P<0.05$ was considered significantly different.

\section{RESULTS}

\section{Rg3 Promotes Cell Growth and Proliferation}

Previous studies have demonstrated that $\mathrm{Rg} 3$ at concentrations of more than $100 \mu \mathrm{M}$ inhibits cell growth, but such high concentrations are difficult to achieve in vivo. To evaluate how $\mathrm{Rg} 3$ affects cell growth at a concentration that is tenable in vivo, we treated HeLa cells with different concentrations of $\mathrm{Rg} 3$ and examined cell growth and proliferation (Figure 1). We found that while $\operatorname{Rg} 3$ inhibited cell proliferation at $50 \mu \mathrm{M}$, surprisingly, at concentrations lower than $50 \mu \mathrm{M}, \mathrm{Rg} 3$ stimulated cell proliferation in a concentration -dependent manner (Figure 1B). We further examined the effect of Rg3 on HeLa cell growth using a CCK8 assay (Figure 1C). As expected, in the presence of Rg3 at a concentration lower than $50 \mu \mathrm{M}$, the number of cells increased at $24 \mathrm{~h}$. This result demonstrated that Rg3 induces HeLa cell proliferation in a dose-dependent manner.

\section{Rg3 Activates the mTORC1 and ERK1/2 Signaling Pathways}

To determine the mechanism by which $\mathrm{Rg} 3$ stimulates cell growth, we examined several major growth-promoting signaling pathways in $\mathrm{Rg} 3$ treated cells. We found that $\mathrm{Rg} 3$ treatment increased mTORC1-dependent phosphorylation of $\mathrm{S} 6$ in a dose-dependent manner that peaked at the concentration of $20 \mu \mathrm{M}$ (Figures 2A,C). However, Rg3 had no obvious effect 

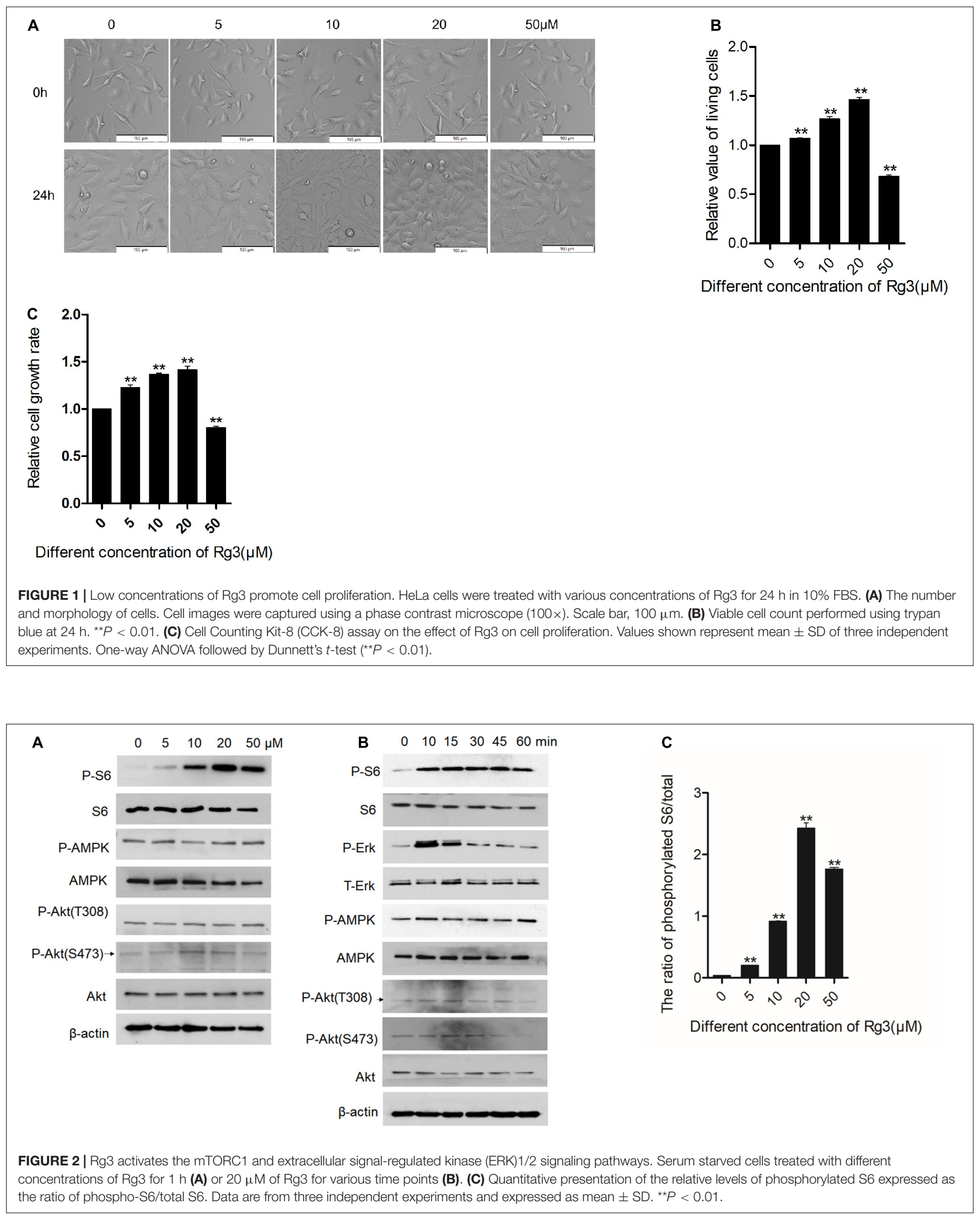

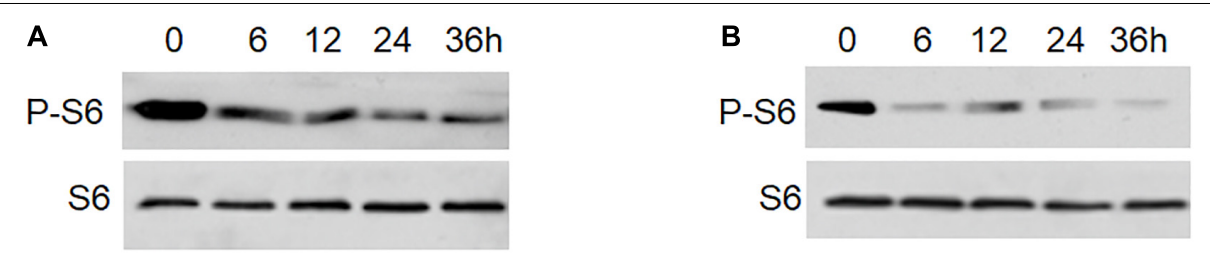

FIGURE 3 | Rg3 attenuates dephosphorylation of S6 upon serum withdrawal. (A) Cells were treated with Rg3 (A) or vehicle control (B) for 30 min followed by serum withdrawal. The levels of phosphorylated S6 and total S6 at indicated time points after the serum withdrawal were determined by Western blotting.

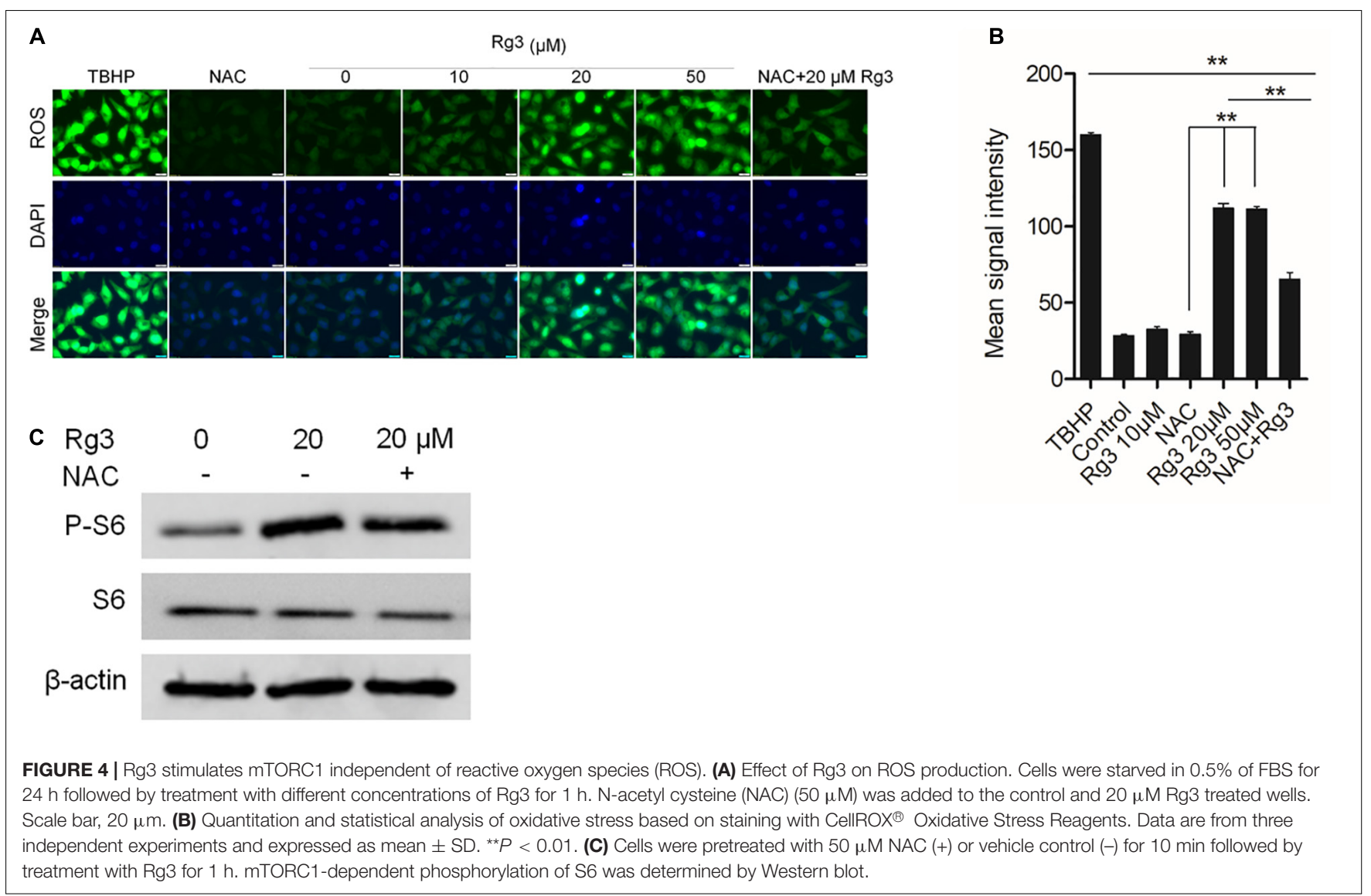

on activation-dependent phosphorylation of AKT and AMPK (Figure 2A), suggesting that $\mathrm{Rg} 3$ activated mTORC1 through an unconventional mechanism. Analysis of the time-dependent activation of mTORC1 revealed that $\mathrm{Rg} 3$ was able to activate mTORC1 within $10 \mathrm{~min}$ after the cells were exposed to the drug (Figure 2B). We also observed a transient activation of ERK1/2, which peaked at $10 \mathrm{~min}$ and then returned to basal level (Figure 2B).

\section{Rg3 Attenuates Dephosphorylation of S6 Upon Serum Withdrawal}

To further determine whether Rg3 stimulates the level of phosphorylated S6 depends on the presence of serum, cells pretreated with or without $\mathrm{Rg} 3$ were starved for serum. As showed in the Figure 3B, in cells without Rg3 treatment, the level of phosphorylated S6 decreased rapidly upon serum withdrawal.
However, the change of phosphorylated S6 was not obvious in the Rg3 treated cells (Figure 3A).

\section{Rg3 Stimulates mTORC1 Independent of ROS}

The above results indicated that $\mathrm{Rg} 3$ affects mTORC1 independent of AKT and AMPK, We further examined if ROS, which stimulates mTORC1 activity at low concentrations (Martin et al., 2013), mediates the effect of $\mathrm{Rg} 3$ on the activation of mTORC1. We first evaluated whether Rg3 stimulates ROS production. We found that in comparison with vehicle-treated cells, cells treated with $\mathrm{Rg} 3$ at a concentration higher than $10 \mu \mathrm{M}$ exhibited a strong accumulation of ROS (Figures 4A,B). Rg3 induced increase in ROS was blocked by treatment with the reducing agent, NAC, However, NAC had no significant effect on the P-S6 level stimulated by $\operatorname{Rg} 3$ 
A

0

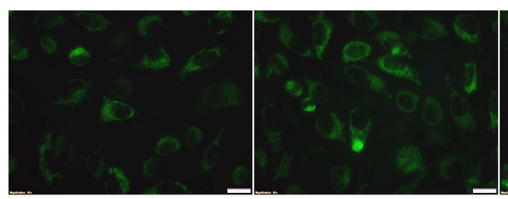

10
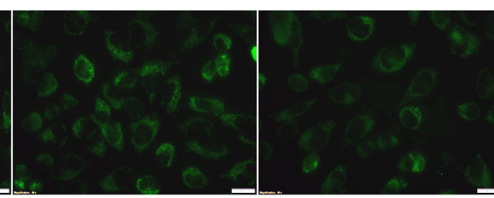

C

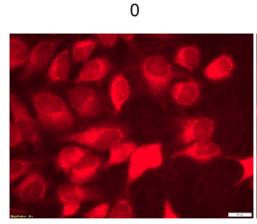

10

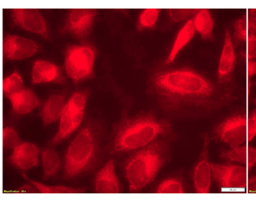

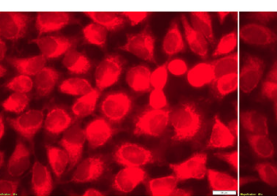

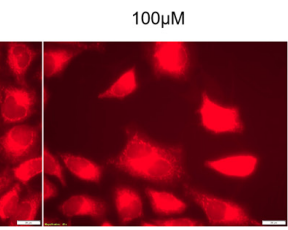

B

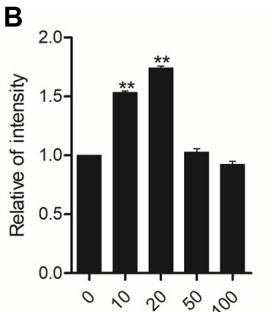

Different concentration of $\operatorname{Rg} 3(\mu \mathrm{M})$

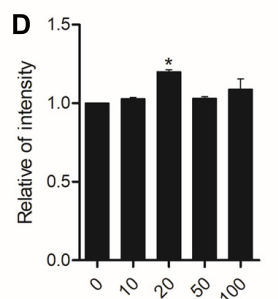

Different concentration of $\operatorname{Rg} 3(\mu \mathrm{M})$

FIGURE 5 | Rg3 promotes mitochondrial biogenesis and function. Cells were treated with different concentrations of Rg3 for $24 \mathrm{~h}$. (A) Cells were stained with $150 \mathrm{nM}$ Mitotracker Green FM for 30 min and imaged with fluorescent microscopy. Scale bar, $100 \mu \mathrm{m}$. (B) Quantitative presentation of the fluorescent intensity on mitochondria shown in panel (A). Data were collected from three independent experiments and expressed as mean \pm SD. ${ }^{* *} P<0.01$ (C) Cells were stained with MitoTracker Red CMXRos (100 nM) for 40 min and imaged with fluorescent microscopy. Scale bar, $100 \mu \mathrm{m}$. (D) Quantitative presentation of the fluorescent intensity of mitochondria shown in panel (C). Data were collected from three independent experiments and expressed in mean \pm SD. ${ }^{\star} P<0.05$.

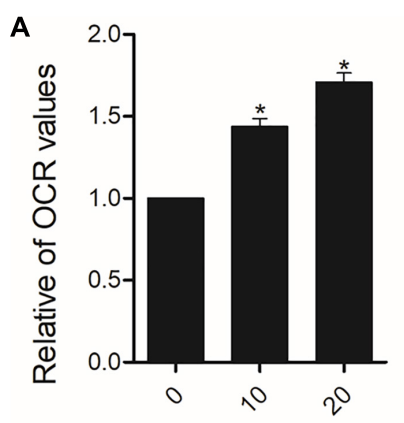

Different concentration of $\operatorname{Rg} 3(\mu \mathrm{M})$

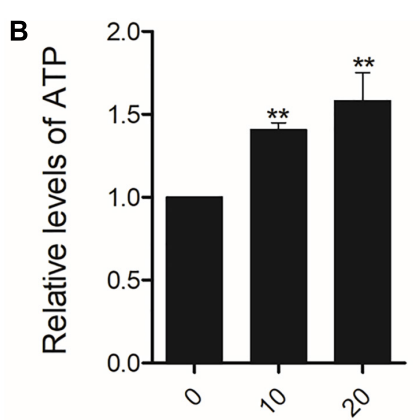

Different concentration of $\operatorname{Rg} 3(\mu \mathrm{M})$

FIGURE 6 | Rg3 increases the basic oxygen consumption and ATP of cells. Cells were treated with Rg3 for $24 \mathrm{~h}$ with $10 \%$ FBS. (A) The basal oxygen consumption rate (OCR) value were obtained using a Seahorse Extracellular Flux Analyzer. ${ }^{\star} P<0.05$. (B) the levels of ATP in the treated cells was measured. The data were from three independent experiments and expressed in mean $\pm \mathrm{SD}$. The differences between the control and Rg3 treated samples. ${ }^{\star \star} P<0$. 01.

(Figure 4C), suggesting that the activation of mTORC1 by Rg3 was independent of ROS.

\section{Rg3 Promotes Mitochondrial Biogenesis and Function}

Next, we examined the effect of $\mathrm{Rg} 3$ on the biogenesis of mitochondria and their function. We used a membrane potential$(\Delta \psi \mathrm{m})$-independent mitochondrial stains, MitoTracker Green FM, to measure the number of mitochondria and $\Delta \psi$ m-dependent dye MitoTracker Red CMXRos to monitor mitochondrial integrity. We found that $\mathrm{Rg} 3$ at concentrations lower than $50 \mu \mathrm{M}$ dose-dependently increased the number of mitochondria. However, the drug had an opposite effect at higher concentrations (Figures 5A,B). Rg3 at $20 \mu \mathrm{M}$ also caused a small but significant increase in mitochondrial membrance potential(Figures 5C,D), suggesting that the drug was able to affect mitochondrial activity. Collectively, these findings demonstrated that $\operatorname{Rg} 3$ can increase the number of mitochondria and affect the activity of mitochondria.

\section{Rg3 Increases the Basic Oxygen Consumption and ATP Generation}

Oxygen consumption rate is a measure of the cellular respiration and mitochondrial function. Using a Seahorse Extracellular Flux Analyzer, we monitored the cellular OCR of cells treated with Rg3 in real time. We found that Rg3 treatment significantly increased the OCR of the cells (Figure 6A). Similarly, $\mathrm{Rg} 3$ also increased the levels of ATP in the treated cells (Figure 6B). These results 
indicated that $\operatorname{Rg} 3$ can increase the basic oxygen consumption and ATP production.

\section{DISCUSSION}

As a ginsenosides monomer, $\mathrm{Rg} 3$ has been reported to be beneficial for the treatment of various cancers t. Previous studies indicate that $\operatorname{Rg} 3$ has potential as a chemo preventive agent or adjuvant treatment. In vitro studies have shown that $\mathrm{Rg} 3$ at high concentrations $(>100 \mu \mathrm{M})$ is able to inhibit cancer cell proliferation and migration (Zhao et al., 2019). However, Rg3 has poor oral bioavailability mainly because of its extensive presystemic metabolism and poor membrane permeability (Jia et al., 2009). Thus, it is very difficult to achieve the concentration of $\mathrm{Rg} 3$ in vivo required for inhibiting cancer cell growth. Therefore, further in vivo studies are required to elucidate the beneficial effects of Rg3 in the treatment of tumors.

In the present study, we show that $\operatorname{Rg} 3$ at low concentrations is able to stimulate, instead of inhibiting, cell growth. We find that $\mathrm{Rg} 3$ has an immediate effect on activating the mTORC1 and MAPK signaling pathways. Surprisingly, Rg3 does not affect the activities of AKT and AMPK, two upstream regulators of mTORC1 that, respectively, channel growth factor and energy signals to mTORC1. This observation suggests that Rg3 activates mTORC1 independent of the conventional mechanisms. We find that $\mathrm{Rg} 3$ can stimulate cellular ROS production. Although it is possible that $\mathrm{Rg} 3$ activates mTORC1 through increasing ROS levels by inducing, oxidative stress, we consider this possibility unlikely for the following two reasons. First, $\operatorname{Rg} 3$ could activate mTORC1 at low concentrations that had no obvious effects on cellular ROS levels (Figure 2B). Second, pretreating cells with NAC prevented Rg3-induced ROS accumulation but failed to block mTORC1 activation. These observations indicate that Rg3 activates mTORC1 through a novel mechanism. The immediate effect of $\mathrm{Rg} 3$ on mTORC1 activation also indicates that $\mathrm{Rg} 3$ may act on mTORC1 through a direct mechanism.

At the concentrations lower than $50 \mu \mathrm{M}, \mathrm{Rg} 3$ also drastically increases the number of mitochondria (Figure 5B), which is accompanied by an enhancement in oxygen consumption and the intracellular ATP level (Figure 6B). The increase in the number of mitochondria is likely to be a consequence of the Rg3-stimulated mTORC1 activation, which has been previously shown to promote mitochondrial biogenesis (Larsson et al., 1985). The high level of mitochondria is expected to lead to an elevated oxygen consumption and ATP production.

The elevated activity of mTORC1 in $\mathrm{Rg} 3$ treated cells is consistent with the higher rate of cell proliferation. To bolster cellular proliferation and growth, mTORC1 stimulates biosynthetic processes including protein synthesis, and acts as a primary regulator of energy production in mitochondria. The synthesis of proteins is positively correlated with the cell proliferative rate (Larsson et al., 1985). In turn, mitochondrial
ATP production is required to fuel protein synthesis and proliferation (Buttgereit and Brand, 1995; Rolfe and Brown, 1997). The correlation between the elevated mTORC1 activity and increased cell proliferation in $\mathrm{Rg} 3$ treated cells indicates that Rg3 may act through enhancing mTORC1 signaling activities to promote cell growth and survival.

\section{CONCLUSION}

In conclusion, our data suggest that $\mathrm{Rg} 3$ at low concentrations is able to promote cell growth through activation of mTORC1. This effect is in contrast with that of high concentrations of $\mathrm{Rg} 3$, which causes cell death. Given the low bioavailability of $\mathrm{Rg} 3$, it is expected to be difficult to achieve high concentrations of $\mathrm{Rg} 3$ in vivo that could exhibit the same cytotoxic effects in vitro. In this regard, the benefit effects of $\mathrm{Rg} 3$ in cancer treatment may lie on its activity in promoting the recovery of normal cells, such as gastric stem cells and hematopoietic precursor cells after chemotherapies. Therefore, the use of $\operatorname{Rg} 3$ compound as an anticancer agent should be evaluated with caution.

\section{DATA AVAILABILITY STATEMENT}

The original contributions presented in the study are included in the article/Supplementary Material, further inquiries can be directed to the corresponding authors.

\section{AUTHOR CONTRIBUTIONS}

YJ and L-ZL designed this study. WL, S-XZ, DZ, L-HH, and BA carried out most of the experiments and wrote the manuscript. Z-SC, L-LS, and H-FP supervised the experiments, analyzed the results, and proofread the manuscript. All authors have read and agreed with the submitted version of the manuscript.

\section{FUNDING}

This work was supported by the Natural Science Foundation of China (Grant Nos. 82004300, 81973816, and 81973775), the China Postdoctoral Science Foundation (Grant No. 2019 M662877), and the Guangdong Province Basic and Applied Basic Research Fund Project (Grant No. 2019A1515110236).

\section{SUPPLEMENTARY MATERIAL}

The Supplementary Material for this article can be found online at: https://www.frontiersin.org/articles/10.3389/fcell.2021. 730309/full\#supplementary-material

\section{REFERENCES}

Aziz, F., Wang, X., Liu, J., and Yan, Q. (2016). Ginsenoside Rg3 induces FUT4mediated apoptosis in $H$. pylori CagA-treated gastric cancer cells by regulating

SP1 and HSF1 expressions. Toxicol. In Vitro 31, 158-166. doi: 10.1016/j.tiv.2015. 09.025

Buttgereit, F., and Brand, M. D. (1995). A hierarchy of ATP-consuming processes in mammalian cells. Biochem. J. 312(Pt 1), 163-167. doi: 10.1042/bj3120163 
Chang, L., Huo, B., Lv, Y., Wang, Y., and Liu, W. (2014). Ginsenoside Rg3 enhances the inhibitory effects of chemotherapy on esophageal squamous cell carcinoma in mice. Mol. Clin. Oncol. 2, 1043-1046. doi: 10.3892/mco.2014.355

Chen, Z., Wei, X., Shen, L., Zhu, H., and Zheng, X. (2019). 20(S)-ginsenoside$\mathrm{Rg} 3$ reverses temozolomide resistance and restrains epithelial-mesenchymal transition progression in glioblastoma. Cancer Sci. 110, 389-400. doi: 10.1111/ cas. 13881

Guo, X. W., Hu, N. D., Sun, G. Z., Li, M., and Zhang, P. T. (2018). Shenyi capsule () plus chemotherapy versus chemotherapy for non-small cell lung cancer: a systematic review of overlapping meta-analyses. Chin. J. Integr. Med. 24, 227-231. doi: 10.1007/s11655-017-2951-5

He, B. C., Gao, J. L., Luo, X., Luo, J., Shen, J., Wang, L., et al. (2011). Ginsenoside $\mathrm{Rg} 3$ inhibits colorectal tumor growth through the down-regulation of Wnt/Bcatenin signaling. Int. J. Oncol. 38, 437-445. doi: 10.3892/ijo.2010.858

Jia, L., Zhao, Y., and Liang, X. J. (2009). Current evaluation of the millennium phytomedicine- ginseng (II): collected chemical entities, modern pharmacology, and clinical applications emanated from traditional Chinese medicine. Curr. Med. Chem. 16, 2924-2942. doi: 10.2174/092986709788803204

Joo, E. J., Chun, J., Ha, Y. W., Ko, H. J., Xu, M. Y., and Kim, Y. S. (2015). Novel roles of ginsenoside $\mathrm{Rg} 3$ in apoptosis through downregulation of epidermal growth factor receptor. Chem. Biol. Interact. 233, 25-34. doi: 10.1016/j.cbi.2015.03.016

Kaufman, D. W., Kelly, J. P., Rosenberg, L., Anderson, T. E., and Mitchell, A. A. (2002). Recent patterns of medication use in the ambulatory adult population of the United States: the Slone survey. JAMA 287, 337-344. doi: 10.1001/jama. 287.3.337

Kim, B. M., Kim, D. H., Park, J. H., Na, H. K., and Surh, Y. J. (2013). Ginsenoside $\mathrm{Rg} 3$ induces apoptosis of human breast cancer (MDA-MB-231) cells. J. Cancer Prev. 18, 177-185. doi: 10.15430/jcp.2013.18.2.177

Kim, B. M., Kim, D. H., Park, J. H., Surh, Y. J., and Na, H. K. (2014). Ginsenoside $\mathrm{Rg} 3$ inhibits constitutive activation of NF- $\kappa \mathrm{B}$ signaling in human breast cancer (MDA-MB-231) cells: ERK and Akt as potential upstream targets. J. Cancer Prev. 19, 23-30. doi: 10.15430/jcp.2014.19.1.23

Kim, H. S., Lee, E. H., Ko, S. R., Choi, K. J., Park, J. H., and Im, D. S. (2004). Effects of ginsenosides $\mathrm{Rg} 3$ and $\mathrm{Rh} 2$ on the proliferation of prostate cancer cells. Arch. Pharm. Res. 27, 429-435. doi: 10.1007/bf02980085

Larsson, O., Zetterberg, A., and Engström, W. (1985). Cell-cycle-specific induction of quiescence achieved by limited inhibition of protein synthesis: counteractive effect of addition of purified growth factors. J. Cell. Sci. 73, 375-387.

Lee, S. Y., Kim, G. T., Roh, S. H., Song, J. S., Kim, H. J., Hong, S. S., et al. (2009). Proteomic analysis of the anti-cancer effect of 20S-ginsenoside Rg3 in human colon cancer cell lines. Biosci. Biotechnol. Biochem. 73, 811-816. doi: $10.1271 /$ bbb. 80637

Li, J., and Qi, Y. (2019). Ginsenoside Rg3 inhibits cell growth, migration and invasion in Caco-2 cells by downregulation of lncRNA CCAT1. Exp. Mol. Pathol. 106, 131-138. doi: 10.1016/j.yexmp.2019.01.003

Lu, P., Su, W., Miao, Z. H., Niu, H. R., Liu, J., and Hua, Q. L. (2008). Effect and mechanism of ginsenoside $\mathrm{Rg} 3$ on postoperative life span of patients with nonsmall cell lung cancer. Chin. J. Integr. Med. 14, 33-36. doi: 10.1007/s11655-0079002-6

Martin, M. V., Distéfano, A. M., Zabaleta, E. J., and Pagnussat, G. C. (2013). New insights into the functional roles of reactive oxygen species during embryo sac development and fertilization in Arabidopsis thaliana. Plant Signal. Behav. 8:e25714. doi: 10.4161/psb.25714

Pan, L., Zhang, T., Sun, H., and Liu, G. (2019). Ginsenoside Rg3 (Shenyi Capsule) combined with chemotherapy for digestive system cancer in China: a meta-analysis and systematic review. Evid. Based Complement Alternat. Med. 2019:2417418. doi: 10.1155/2019/2417418

Rolfe, D. F., and Brown, G. C. (1997). Cellular energy utilization and molecular origin of standard metabolic rate in mammals. Physiol. Rev. 77, 731-758. doi: 10.1152/physrev.1997.77.3.731

Shi, Y., Wang, H., Zheng, M., Xu, W., Yang, Y., and Shi, F. (2020). Ginsenoside Rg3 suppresses the NLRP3 inflammasome activation through inhibition of its assembly. FASEB J. 34, 208-221. doi: 10.1096/fj.201901537R

Wang, J., Tian, L., Khan, M. N., Zhang, L., Chen, Q., Zhao, Y., et al. (2018). Ginsenoside Rg3 sensitizes hypoxic lung cancer cells to cisplatin via blocking of
NF-кB mediated epithelial-mesenchymal transition and stemness. Cancer Lett. 415, 73-85. doi: 10.1016/j.canlet.2017.11.037

Wang, J. H., Nao, J. F., Zhang, M., and He, P. (2014). 20(s)-ginsenoside Rg3 promotes apoptosis in human ovarian cancer HO-8910 cells through PI3K/Akt and XIAP pathways. Tumour Biol. 35, 11985-11994. doi: 10.1007/s13277-0142497-5

Wang, X. J., Zhou, R. J., Zhang, N., and Jing, Z. (2019). 20(S)-ginsenoside Rg3 sensitizes human non-small cell lung cancer cells to icotinib through inhibition of autophagy. Eur. J. Pharmacol. 850, 141-149. doi: 10.1016/j.ejphar.2019.02. 023

Whitworth, J. M., Londoño-Joshi, A. I., Sellers, J. C., Oliver, P. J., Muccio, D. D., Atigadda, V. R., et al. (2012). The impact of novel retinoids in combination with platinum chemotherapy on ovarian cancer stem cells. Gynecol. Oncol. 125, 226-230. doi: 10.1016/j.ygyno.2011.12.425

Xie, H-T., Wang, G-J., Sun, J. G., Tucker I., Zhao, X-C., Xie, Y-Y., et al. (2005). High performance liquid chromatographic-mass spectrometric determination of ginsenoside $\mathrm{Rg} 3$ and its metabolites in rat plasma using solid-phase extraction for pharmacokinetic studies. J. Chromatogr. B. Analyt. Technol. Biomed. Life Sci. 818, 167-173. doi: 10.1016/j.jchromb.2004.12.028

Yang, J., Yuan, D., Xing, T., Su, H., Zhang, S., Wen, J., et al. (2016). Ginsenoside Rh2 inhibiting HCT116 colon cancer cell proliferation through blocking PDZbinding kinase/T-LAK cell-originated protein kinase. J. Ginseng Res. 40, 400408. doi: 10.1016/j.jgr.2016.03.007

Yang, X., Zou, J., Cai, H., Huang, X., Yang, X., Guo, D., et al. (2017). Ginsenoside $\mathrm{Rg} 3$ inhibits colorectal tumor growth via down-regulation of C/EBP $\beta / N F-\kappa B$ signaling. Biomed. Pharmacother. 96, 1240-1245. doi: 10.1016/j.biopha.2017.11. 092

Yuan, H. D., Quan, H. Y., Zhang, Y., Kim, S. H., and Chung, S. H. (2010). 20(S)Ginsenoside Rg3-induced apoptosis in HT-29 colon cancer cells is associated with AMPK signaling pathway. Mol. Med. Rep. 3, 825-831. doi: 10.3892/mmr. 2010.328

Yuan, Z., Jiang, H., Zhu, X., Liu, X., and Li, J. (2017). Ginsenoside Rg3 promotes cytotoxicity of Paclitaxel through inhibiting NF- $\kappa \mathrm{B}$ signaling and regulating $\mathrm{Bax} / \mathrm{Bcl}-2$ expression on triple-negative breast cancer. Biomed. Pharmacother. 89, 227-232. doi: 10.1016/j.biopha.2017.02.038

Zhang, F., Li, M., Wu, X., Hu, Y., Cao, Y., Wang, X., et al. (2015). 20(S)-ginsenoside $\mathrm{Rg} 3$ promotes senescence and apoptosis in gallbladder cancer cells via the p53 pathway. Drug Des. Devel. Ther. 9, 3969-3987. doi: 10.2147/DDDT.S84527

Zhao, L., Shou, H., Chen, L., Gao, W., Fang, C., and Zhang, P. (2019). Effects of ginsenoside $\operatorname{Rg} 3$ on epigenetic modification in ovarian cancer cells. Oncol. Rep. 41, 3209-3218. doi: 10.3892/or.2019.7115

Zhong, M., Zhao, X., Li, J., Yuan, W., Yan, G., Tong, M., et al. (2016). Tumor suppressor folliculin regulates mTORC1 through primary cilia. J. Biol. Chem. 291, 11689-11697. doi: 10.1074/jbc.M116.719997

Zhou, B., Yan, Z., Liu, R., Shi, P., Qian, S., Qu, X., et al. (2016). Prospective study of transcatheter arterial chemoembolization (TACE) with ginsenoside Rg3 versus TACE alone for the treatment of patients with advanced hepatocellular carcinoma. Radiology 280, 630-639. doi: 10.1148/radiol.2016150719

Conflict of Interest: The authors declare that the research was conducted in the absence of any commercial or financial relationships that could be construed as a potential conflict of interest.

Publisher's Note: All claims expressed in this article are solely those of the authors and do not necessarily represent those of their affiliated organizations, or those of the publisher, the editors and the reviewers. Any product that may be evaluated in this article, or claim that may be made by its manufacturer, is not guaranteed or endorsed by the publisher.

Copyright (C) 2021 Liu, Zhang, Ai, Pan, Zhang, Jiang, Hu, Sun, Chen and Lin. This is an open-access article distributed under the terms of the Creative Commons Attribution License (CC BY). The use, distribution or reproduction in other forums is permitted, provided the original author(s) and the copyright owner(s) are credited and that the original publication in this journal is cited, in accordance with accepted academic practice. No use, distribution or reproduction is permitted which does not comply with these terms. 